\title{
Elevated ZNF703 Protein Expression Is an Independent Unfavorable Prognostic Factor for Survival of the Patients with Head and Neck Squamous Cell Carcinoma
}

\author{
Hang Yang, ${ }^{1,2}$ Wen-Qi Jiang, ${ }^{1,2}$ Ye Cao, ${ }^{1,3}$ Yong-An Sun, ${ }^{4}$ Jing Wei, ${ }^{5}$ Xin An, ${ }^{1,2}$ \\ Ying-Chun Zhang, ${ }^{1,6}$ Ming Song, ${ }^{1,7}$ Shu-Sen Wang, ${ }^{1,2}$ Zhong-Yu Yuan, ${ }^{1,2}$ Rou-Jun Peng, ${ }^{1,2}$ \\ Tan-Huan Chen, ${ }^{8}$ Li-Ren Li, ${ }^{1,9}$ and Yan-Xia Shi ${ }^{1,2}$ \\ ${ }^{1}$ Sun Yat-Sen University Cancer Center, State Key Laboratory of Oncology in South China, Collaborative Innovation Center for \\ Cancer Medicine, Guangzhou 510060, China \\ ${ }^{2}$ Department of Medical Oncology, Sun Yat-Sen University Cancer Center, Guangzhou 510060, China \\ ${ }^{3}$ Clinical Trial Center, Sun Yat-Sen University Cancer Center, Guangzhou 510060, China \\ ${ }^{4}$ Department of Neurology, Peking University First Hospital, Beijing 100034, China \\ ${ }^{5}$ Department of Medical Oncology, Guangxi Traditional Chinese Medical University, Ruikang Hospital, Nanning 530011, China \\ ${ }^{6}$ Department of Pathology, Sun Yat-Sen University Cancer Center, Guangzhou 510060, China \\ ${ }^{7}$ Department of Head and Neck Surgery, Sun Yat-Sen University Cancer Center, Guangzhou 510060, China \\ ${ }^{8}$ Department of Oncology, Huizhou Municipal Central Hospital, Huizhou 516001, China \\ ${ }^{9}$ Department of Colorectal Surgery, Sun Yat-Sen University Cancer Center, Guangzhou 510060, China
}

Correspondence should be addressed to Li-Ren Li; lilr@sysucc.org.cn and Yan-Xia Shi; shiyx@sysucc.org.cn

Received 11 February 2015; Accepted 15 April 2015

Academic Editor: Silvia Persichilli

Copyright (C) 2015 Hang Yang et al. This is an open access article distributed under the Creative Commons Attribution License, which permits unrestricted use, distribution, and reproduction in any medium, provided the original work is properly cited.

\begin{abstract}
Aim. Data from The Cancer Genome Atlas (TCGA) show that the ZNF703 gene amplifies and overexpresses in head and neck squamous cell carcinomas (HNSCC). However, the clinical relevance of this observation in HNSCC is unclear. The purpose of this study was to clarify the expression of ZNF703 protein and its prognostic effect on HNSCC. Methods. Two hundred ten HNSCC patients from Sun Yat-Sen University Cancer Center with complete survival follow-up were included in this study. Tumor samples from primary sites were collected. The expression of the ZNF703 protein was tested by immunohistochemistry (IHC). Results. The high expression of ZNF703 in HNSCC tumor tissues was significantly higher than that of the matched noncancerous tissues (48.6\% versus $11.6 \%, P<0.001$ ). ZNF703 overexpression was correlated with tumor position (laryngeal carcinoma) and recurrence (all $P<$ $0.05)$. Multivariate analysis revealed that ZNF703 protein overexpression was an independent prognostic factor $(P=0.022$, hazard ratio $=1.635,95 \%$ CI 1.073-2.493) in HNSCC patients. Conclusion. ZNF703 overexpression is associated with adverse prognosis in HNSCC, which might be a novel biomarker of HNSCC.
\end{abstract}

\section{Introduction}

Head and Neck Squamous Cell Carcinoma (HNSCC) is the sixth most common malignancy in the world, with approximately 600,000 newly diagnosed cases per year $[1,2]$. HNSCC is a group of fairly heterogeneous tumors that can occur from the base of the cranium to the clavicles. On the basis of anatomic sites, HNSCC can be divided into various types; the paranasal sinuses, nasal cavities, nasopharynx, orbits, oral cavity, oropharynx, hypopharynx, and larynx make up 90\% of HNSCC and the 5-year survival rate of all HNSCC is approximately $50 \%$ [3]. Patients diagnosed at an early stage can have a superior quality of life and life expectancy with surgery alone [3]. The majority of patients are diagnosed at an advanced stage, whose prognosis is extremely poor [4]. For patients in stage IV, the long-term survival of HNSCC 
patients is only approximately 15 months [5]; the high recurrence rate highlights the need for a cure. Despite advances in diagnosis and treatment, the survival rates for many types of HNSCC have improved little over the past forty years [2, 3]. Therefore, a deeper understanding of HNSCC pathogenesis is needed to promote the development of improved therapeutic approaches.

In recent years, the development of high-throughput sequencing technology provides the best opportunity for exploring fundamental tumorigenic mechanisms. According to The Cancer Genome Atlas (TCGA) database, which included 4000 cases and more than 20 tumor types, ZNF703 is one of the most frequently altered genes in the pan-cancer group. In HNSCC, the incidence of an increased ZNF703 copy number was $28 \%$, and the high-level of gene amplification accounted for 7.0\% [http://www.cbioportal.org/publicportal/].

The zinc finger protein 703 (also known as ZPO1, ZEPPO1) gene is located on chromosome arm $8 \mathrm{p} 12$. The ZNF703 protein belongs to the NET (Noc/Nlz, Elbow, and Tlp-1) family, which plays an important role in the embryonic development of zebrafish [6] and Drosophila [7]. It has been shown that ZNF703 gene amplification stimulates migration and proliferation while reducing cell to cell adhesion [8-10] and is speculated to be associated with poorer outcomes [912] in breast cancer. Two studies from China also discovered that ZNF703 acts as an oncogene that accelerates malignant progression in gastric cancer [13] and is associated with worse prognosis in colorectal cancer patients [14]. As a consequence, we propose that ZNF703 may also play an important role in the pathogenesis of HNSCC. However, we are not aware of any reports about ZNF703 in HNSCC until now. Thus, we conducted this retrospective study to explore the expression of ZNF703 protein and its clinical relevance in HNSCC.

\section{Materials and Methods}

2.1. Patients and Tissue Specimens. A total of 210 HNSCC patients (160 male and 50 female) between January 2001 and December 2008 with complete survival follow-up documents and available tumor samples were enrolled in our study. All patients had undergone surgery at Sun Yat-Sen University Cancer Center, Guangzhou. The exclusion criteria were as follows: (i) accepted neoadjuvant radiotherapy and/or chemotherapy before surgery, (ii) having distant metastasis at the time of diagnosis (stage IVc), (iii) incomplete records, (iv) having other malignant tumors at any time during the treatment and follow-up, (v) with severe complications before treatment. The TNM stage was reclassified on the basis of the American Joint Committee TNM Staging system (7th ed., 2010). All patients were followed up until April 2014. Two hundred ten paraffin-embedded specimen blocks and 43 matched adjacent noncancerous tissue blocks (as control group) were obtained from the pathology department of Sun Yat-Sen University Cancer Center. All the slides were reviewed to reconfirm the diagnosis by two independent pathologists. All works were conducted in accordance with the Declaration of Helsinki (1964). Written and informed consent was obtained from every patient, and the study was approved by the ethics review board of Sun Yat-Sen University Cancer Center. Local or distant metastasis was confirmed by biopsy or radiological examination.

2.2. Immunohistochemistry (IHC) and Assessment. The paraffin sections ( $4 \mathrm{~mm}$ thick) were dewaxed and rehydrated, and endogenous peroxidase was first blocked with $0.3 \%$ $\mathrm{H}_{2} \mathrm{O}_{2}$ methanol. For antigen retrieval, the tissue slides were blocked in the $0.01 \mathrm{M}$ citrate buffer ( $\mathrm{pH}$ 6.0) in a microwave oven for $30 \mathrm{~min}$. Nonspecific binding was inhibited with normal goat serum for $30 \mathrm{~min}$. The primary ZNF703 antibody (ab155210, 1:400, Abcam, Cambridge, MA, USA) was used, and slides were put in a moist chamber overnight at $4^{\circ} \mathrm{C}$. Subsequently, the slides were incubated with biotinylated secondary antibody for $30 \mathrm{~min}$ at $37^{\circ} \mathrm{C}$. Horseradish peroxidase was subsequently applied. Finally, Meyer's hematoxylin was used for nucleus counterstaining. The negative control was obtained by omitting the primary antibody.

All immunostaining slides were judged by two independent pathologists without knowledge of the clinic-pathologic information. The specific intensities of staining were identified as follows: $0=$ none; $1=$ weak; $2=$ moderate; $3=$ strong. The proportion scores were as follows: $0=$ none; $1=$ $1-10 \% ; 2=11-50 \% ; 3=51-80 \% ; 4=>81 \%$. The ZNF703 immunoreactive score for each tissue was calculated by multiplying the score of the staining intensity by that of the percentage of positive cells. If the discrepancy between the two pathologists' immunoreactive score was larger than 6 , the tissue section was reevaluated by the two pathologists face to face.

2.3. Cut-Off Score Selection. The median computed value of ZNF703 protein staining was 3, with a range of 0-12. The receiver operating characteristic (ROC) curve for the ZNF703 scope were plotted to select the potential cut-off score (Figure 1$)$. The area under the curve was $0.629(P=$ $0.001,95 \%$ confidence interval $=0.553-0.705)$. As the optimal cut-off score, 3.5 point on the ROC curve could maximize the Youden Index $[1.178, \mathrm{YI}=$ sensitivity $(0.544)+$ specificity $(0.633)]$. Thus, cases were divided into "low expression" (Allred score $\leq 3$ ) and "high expression" (Allred score $>3$ ) [15].

2.4. Statistical Analysis. The SPSS 19.0 software was used for statistical analyses. A receiver operating characteristic (ROC) curve and the median was used to determine the cut-off value to distinguish high or low ZNF703 expression. The relationship between clinic-pathological characteristics and ZNF703 expression was assessed by a $\chi^{2}$-test. Overall survival time was defined as the duration between the time of disease diagnosis and the time of death. The Kaplan-Meier method was used for survival analysis. The Log-Rank test was conducted for differential survival analysis. Additionally, the Cox test was performed for multivariate analysis. The statistical tests were two-sided probability tests. Statistical significance was defined as less than 0.05 . 


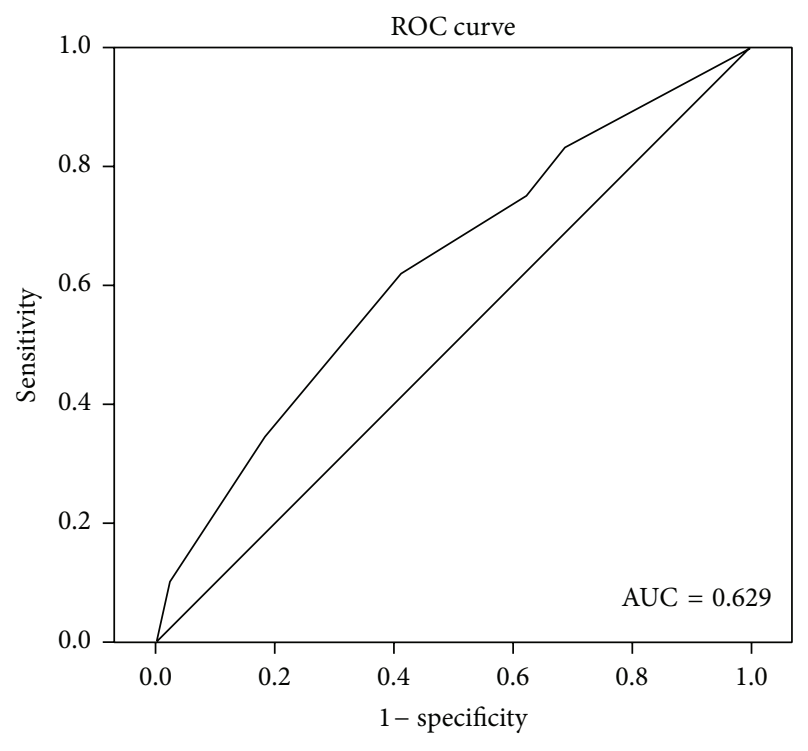

FIGURE 1: Selection of the cut-off score for positive expression of ZNF703 by ROC analysis. The area under the curve was 0.629 , the point 3.5 could maximize the Youden Index [1.178, YI = sensitivity $(0.544)+$ specificity $(0.633)]$.

\section{Results}

3.1. Clinical Features of Patients. The clinical features of the 210 patients are listed in Table 1. The TNM stage of the patients ranged from stage I to IVb. The median age was 53.5 years (range 25-86 years). All the patients had undergone complete excision with or without unilateral/bilateral neck dissection and accepted adjuvant radiotherapy according to the NCCN guideline. During the follow-up period, there were $132(62.9 \%)$ local and distant recurrence events and 90 patients (42.9\%) died. The median overall survival time (OS) was 80.4 (2.6-169.6) months, The 1-, 3-, and 5-year survival rates were $91.4 \%, 70.4 \%$, and $64.1 \%$, respectively. The diseasefree survival (DFS) was in a range of 0.7-139.8 months, and the 1-, 3-, and 5-year disease-free survival rates were $63.8 \%$, $46.7 \%$, and $41.8 \%$, respectively. Among the patients with recurrence, eighty-nine $(67.4 \%)$ patients died by the end of follow-up, and the 1, 3, and 5-year survival rates were $86.5 \%$, $52.7 \%$, and $43.4 \%$, respectively.

3.2. The Relationship between ZNF703 Expression and Clinicopathological Factors. The ZNF703 protein was located in the cytoplasm and nucleus, but mainly in the nucleus (Figure 2). The overexpression rate of ZNF703 protein was $48.6 \%$ in HNSCC tumor cells, it was significantly higher than that of the adjacent noncancerous squamous epithelium cells (Figure 3, 48.6\% versus $11.6 \%, P<0.001$ ). The overexpression of ZNF703 was related to recurrence and primary tumor site but not related to $\mathrm{T}$ stage, $\mathrm{N}$ stage, age, gender, $\mathrm{BMI}$, differentiation, and complications (Table 1).

3.3. The Relationship between ZNF703 Expression and Prognosis. Comparing to the ZNF703 low expression group, the ZNF703 high expression group had a significantly higher recurrence rate $(71.0 \%$ versus $56.4 \%, P=0.03)$ and mortality (52.7\% versus $35.0 \%, P=0.01)$. The mean OS and DFS in the ZNF703 overexpression group were 89.3 months (95\% CI 74.3-104.3) and 43.7 months (95\% CI 34.8-52.6), respectively. The 5-year OS and DFS rates were $58.9 \%$ and $34.2 \%$, respectively, whereas in the ZNF703 low level group, the mean OS and DFS times were 117.7 months (95\% CI 105.1-130.4) and 71.1 months (95\% CI 59.7-82.5), respectively, and the 5year OS and DFS data were 68.2 and $46.9 \%$, respectively. In univariate survival analysis, a high ZNF703 protein level was an important prognostic factor for shorter overall survival $(P=0.009)$ and DFS $(P=0.019)$ (Table 2, Figure 4). It was also observed that male patients, patients who consumed tobacco and alcohol, and patients with advanced stage disease, complications, or poorer differentiation had an apparently shorter OS. Meanwhile, poorer differentiation, advanced stage disease, without neck dissection or adjuvant radiotherapy and laryngeal cancer were all associated with shorter DFS (Table 2). In the multivariate Cox proportional hazards model analysis, only ZNF703 overexpression $(P=$ 0.022 , hazard ratio $=1.635,95 \%$ CI 1.073-2.493), advanced stage, complications, and alcohol consumption were independent prognostic factors in HNSCC patients (Table 3).

\section{Discussion}

A variety of genetic changes have been described previously in HNSCC, including frequent chromosome $8 \mathrm{p}$ amplification [16-21]. It has also been identified that amplifications at $8 \mathrm{p} 12$ occurred frequently in multiple cancer types. This was suggestive of the presence of an important oncogene in this region, and numerous studies have been carried out to identify the driver gene on $8 \mathrm{p} 12$.

There are five genes in this area: ERLIN2, $P R$ OSC, BRF2, RAB11FIP1, and ZNF703 [22-24]. Recent research showed that ZNF703 is amplified in approximately $15 \%$ of breast tumors [23, 25-27], which is only less than HER2 and cyclin D1 (CCND1) $[26,28,29]$, especially in Luminal B molecular subtypes [9-11, 29]; thus, more attention has been focused on ZNF703.

In the TCGA database of HNSCC, the amplification rate of the ZNF703 gene is 7\% [http://www.cbioportal.org/publicportal]. Our results also showed that the expression level of ZNF703 protein was significantly higher in HNSCC than in the adjacent noncancerous squamous epithelial cells, the overexpression rates were $48.6 \%$ and $11.6 \%$, respectively $(P<$ 0.001). Furthermore, ZNF703 overexpression was observed more frequently in larynx cancer and was correlated with higher risk of recurrence. The 5-year OS rate was 58.9\% and $68.2 \%$ in the high and low expression groups, respectively. All these results indicated that ZNF703 may play a role in the tumorigenesis and metastasis of HNSCC and act as an oncogene.

Overexpression of ZNF703 was also found in other cancers, such as breast cancer, gastric cancer, and colorectal cancer. Zhang et al. reported that the positive expression rate of ZNF703 in early stage breast cancer patient was 91.3\% [12]. 
TABLE 1: Correlations between ZNF703 expression and clinicopathological characteristics of the patients.

\begin{tabular}{|c|c|c|c|}
\hline \multirow{2}{*}{ Parameter } & \multicolumn{2}{|c|}{ ZNF703 expression } & \multirow{2}{*}{$P$ value } \\
\hline & Low (117) & High (93) & \\
\hline Age ( years) & & & 0.420 \\
\hline$\leq 50$ & 52 & 31 & \\
\hline $51-60$ & 34 & 32 & \\
\hline $61-70$ & 24 & 22 & \\
\hline$>70$ & 7 & 8 & \\
\hline Gender & & & 0.093 \\
\hline Male & 84 & 76 & \\
\hline Female & 33 & 17 & \\
\hline $\operatorname{BMI}\left(\mathrm{kg} / \mathrm{m}^{2}\right)$ & & & 0.298 \\
\hline$<20$ & 22 & 24 & \\
\hline 20 to $<25$ & 70 & 46 & \\
\hline$\geq 25$ & 25 & 23 & \\
\hline Tobacco consumption & & & 0.202 \\
\hline No & 53 & 34 & \\
\hline Yes & 64 & 59 & \\
\hline Alcohol consumption & & & 0.208 \\
\hline No & 93 & 67 & \\
\hline Yes & 24 & 26 & \\
\hline Histological differentiation & & & 0.324 \\
\hline Well & 76 & 51 & \\
\hline Moderately & 31 & 31 & \\
\hline Poorly & 10 & 11 & \\
\hline Complications & & & 0.649 \\
\hline No & 96 & 74 & \\
\hline Yes & 21 & 19 & \\
\hline T stage & & & 0.549 \\
\hline $\mathrm{T} 1$ & 68 & 45 & \\
\hline $\mathrm{T} 2$ & 31 & 30 & \\
\hline $\mathrm{T} 3$ & 12 & 13 & \\
\hline $\mathrm{T} 4$ & 6 & 5 & \\
\hline $\mathrm{N}$ stage & & & 0.071 \\
\hline N0 & 87 & 67 & \\
\hline N1 & 19 & 12 & \\
\hline $\mathrm{N} 2$ & 11 & 10 & \\
\hline N3 & 0 & 4 & \\
\hline Cancer stage & & & 0.362 \\
\hline I/II & 80 & 58 & \\
\hline III/IV & 37 & 35 & \\
\hline Tumor position & & & 0.026 \\
\hline Oral cavity & 100 & 68 & \\
\hline Buccal mucosa & 4 & 8 & \\
\hline Floor of mouth & 9 & 3 & \\
\hline Anterior tongue & 70 & 41 & \\
\hline Alveolar ridge & 15 & 14 & \\
\hline Hard palate & 2 & 2 & \\
\hline Larynx & 17 & 25 & \\
\hline Glottis & 12 & 18 & \\
\hline Supraglottic & 5 & 7 & \\
\hline Disease recurrence & & & 0.030 \\
\hline No & 51 & 27 & \\
\hline Yes & 66 & 66 & \\
\hline
\end{tabular}

BMI = body mass index. 


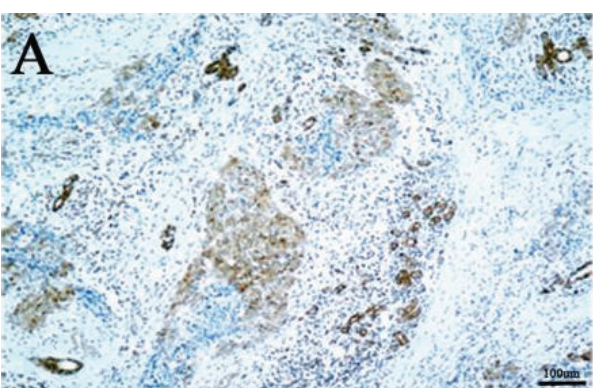

(a)

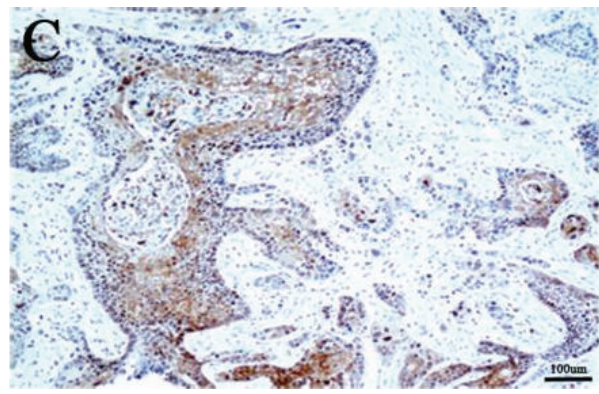

(c)

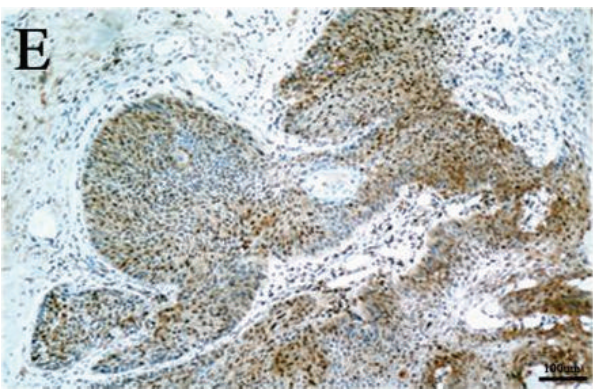

(e)

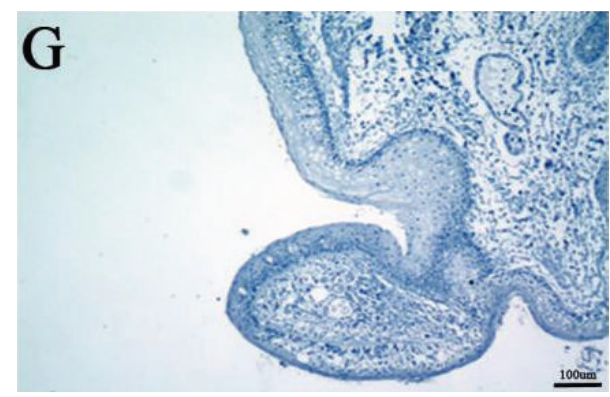

(g)

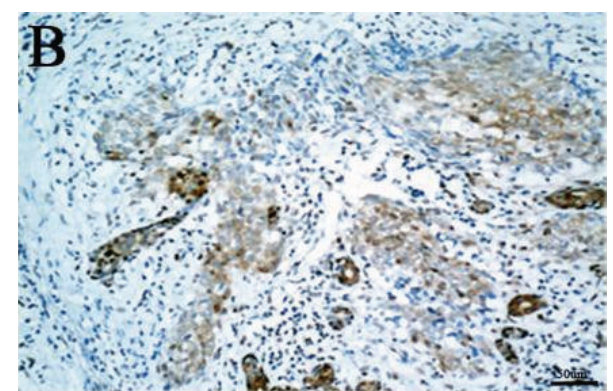

(b)

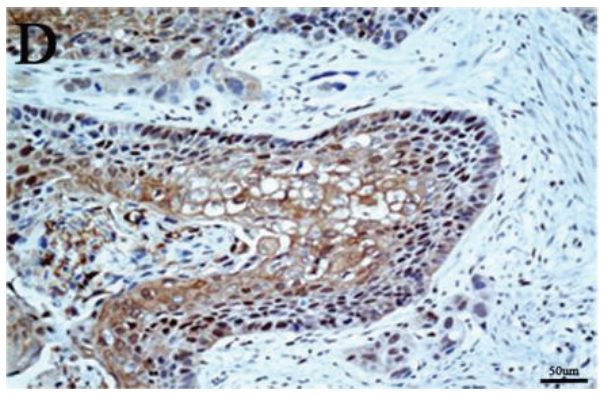

(d)

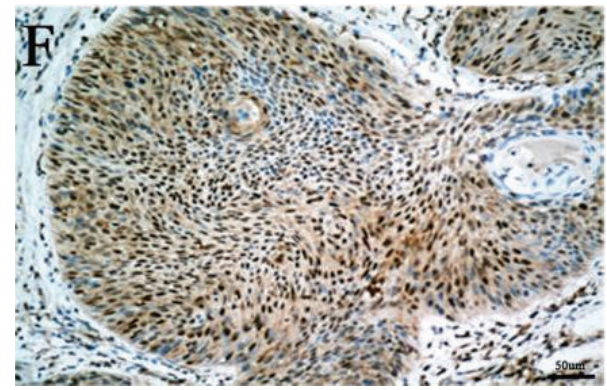

(f)

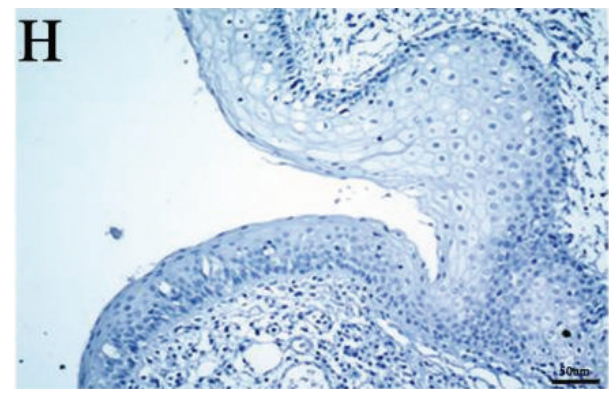

(h)

FiguRE 2: Expression of ZNF703 in tumor species and noncancerous species. ((a), (b)) Weak positivity in tumor cells, mainly stained in cytoplasm (100x and 200x, resp.). ((c), (d)) Moderate positivity in tumor cells, which are located in nucleus and cytoplasm (100x and 200x, resp.). ((e), (f)) Strong positivity in squamous carcinoma cells, mainly shown as nuclear staining. (100x and 200x, resp.). ((g), (h)) No ZNF703 protein staining was present in the noncarcinoma epithelium.

Yang et al. found that the protein was also upregulated in invasive gastric carcinoma tissues [13] and colorectal cancer samples [14]. Interestingly, further studies reported that patients with higher $\mathrm{N}$ stage and high risk of tumor relapse and death had a higher level of ZNF703 expression $(P<0.05)$.
This finding prompted researchers to consider whether the ZNF703 protein is a candidate factor for promoting HNSCC growth, progress and metastasis.

Although there is still no direct report about the pathogenic mechanism of ZNF703 in HNSCC, reports in 


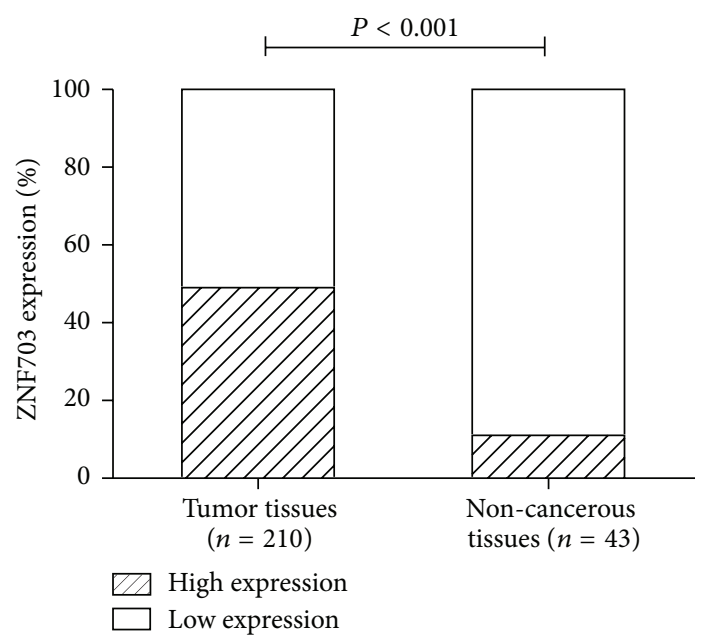

FIGURE 3: The expression of ZNF703 protein is elevated in tumor tissues and noncancerous specimens. The comparison of ZNF703 protein expression evaluated by IHC revealed that the percentage of high-expression cases in tumor tissues was significantly higher than that of the adjacent noncancerous matched tissue $(n=43)$.

other cancer types may help to understand its mechanism. Earlier studies also showed that ZNF703 stimulated mammary epithelial cells toward immortalization rather than differentiation and enhanced the invasive potential of breast cancer cells $[9,10]$. The possible mechanism is thought to be involved in the EMT. The ZNF703 could downregulate E-cadherin and promote metastasis-associated p120-catenin isoform 1 expression. Subsequent data demonstrated that ZNF703 overexpression increased lung metastases in a mouse breast cancer model $[8,30]$. It may also play a role in the activation of stem cell-related gene expression, leading to an increase in cancer stem cells [10]. On the other hand, it was reported to be involved in the activation of the Akt/mTOR signaling pathway, downregulated ERalpha, and the reduction of the antitumor effect of tamoxifen in breast cancer in vitro [12]. Based on these results we suggest that ZNF703 may also play an important role in HNSCC formation and progression.

In our study, we only detected ZNF703 protein expression with IHC, so there is no final conclusion about the status of ZNF703 gene amplification or mRNA levels in HNSCC. However, according to the TCGA database of patient tumor samples and the CCLE database of cell lines and other literature [9], the copy number and gene expression are highly positively correlated, $r=0.72$ [http:// www.cbioportal.org/public-portal].

On the basis of these results, we speculate that ZNF703 could be a valuable independent factor in HNSCC for predicting prognosis as well as a promising biomarker to help design optimal individual treatments.

\section{Conclusion}

On the one hand, our findings suggested that the overexpression of ZNF703 is common in patients with HNSCC and is related to the aggressive clinical course and poor prognosis. Therefore, more aggressive therapy and closer follow-up
TABLE 2: Univariate survival analysis of different parameters in 210 patients with HNSCC.

\begin{tabular}{lcc}
\hline Variables & \multicolumn{2}{c}{$P$ value } \\
\hline Male & $\mathbf{0 . 0 4 8}$ & 0.310 \\
Complications & $\mathbf{0 . 0 4 7}$ & 0.312 \\
Tobacco consumption & $\mathbf{0 . 0 1 3}$ & 0.128 \\
Alcohol consumption & $\mathbf{0 . 0 0 1}$ & 0.087 \\
Age $>70$ or $<45$ & $0.512 / 0.313$ & $0.514 / 0.761$ \\
BMI $<20$ or $\geq 25$ & $0.519 / 0.427$ & $0.975 / 0.518$ \\
Poorer differentiation & $\mathbf{0 . 0 0 5}$ & $\mathbf{0 . 0 0 1}$ \\
Without neck dissection & 0.705 & $\mathbf{0 . 0 0 2}$ \\
ZNF703 high expression & $\mathbf{0 . 0 0 9}$ & $\mathbf{0 . 0 1 9}$ \\
Stage III/IV & $\mathbf{0 . 0 0 0}$ & $\mathbf{0 . 0 0 1}$ \\
Larynx cancer & 0.509 & $\mathbf{0 . 0 0 0}$ \\
Without adjuvant radiotherapy & 0.308 & $\mathbf{0 . 0 3 7}$ \\
\hline
\end{tabular}

should be adopted in patients with ZNF703 overexpression. On the other hand, our findings also provide new clues for further exploration of new treatments in HNSCC. To the best of our knowledge, this is the first report about the expression of ZNF703 in HNSCC. However, due to the retrospective research and small sample size, further prospective clinical study and genomic, cellular function and mechanistic studies are needed to prove our conclusions.

\section{Conflict of Interests}

The authors declare no conflict of interests regarding the publication of this paper. 
TABLE 3: Multivariate analysis of different parameters in 210 patients with HNSCC by Cox proportional hazard mode.

\begin{tabular}{|c|c|c|c|}
\hline \multirow{2}{*}{ Variables } & \multicolumn{3}{|c|}{ OS } \\
\hline & $\mathrm{RR}$ & $95 \% \mathrm{CI}$ & $P$ \\
\hline Male & 0.955 & $0.466-1.955$ & 0.899 \\
\hline Complications & 0.583 & $0.358-0.951$ & 0.031 \\
\hline Tobacco consumption & 0.755 & $0.421-1.352$ & 0.344 \\
\hline Alcohol consumption & 0.574 & $0.358-0.922$ & 0.022 \\
\hline Poor differentiation & 1.245 & $0.798-1.942$ & 0.334 \\
\hline ZNF703 high expression & 1.635 & $1.073-2.493$ & 0.022 \\
\hline Stage III/IV & 2.661 & $1.689-4.190$ & 0.000 \\
\hline \multirow{2}{*}{ Variables } & \multicolumn{3}{|c|}{ DFS } \\
\hline & $\mathrm{RR}$ & $95 \% \mathrm{CI}$ & $P$ \\
\hline Poor differentiation & 1.129 & $0.761-1.674$ & 0.546 \\
\hline Without neck dissection & 1.447 & $0.974-2.149$ & 0.068 \\
\hline Without adjuvantradiotherapy & 1.656 & $1.077-2.546$ & 0.021 \\
\hline ZNF703 high expression & 1.419 & $0.999-2.016$ & 0.050 \\
\hline Stage III/IV & 1.782 & $1.205-2.634$ & 0.004 \\
\hline Larynx cancer & 0.543 & $0.351-0.842$ & 0.006 \\
\hline
\end{tabular}

$\mathrm{RR}=$ relative risk $\mathrm{CI}=$ confidence interval.

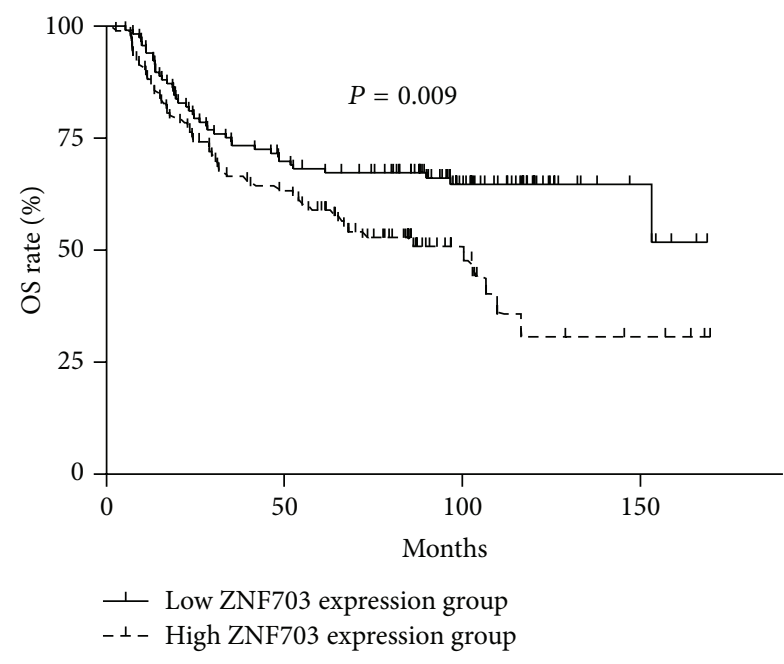

(a)

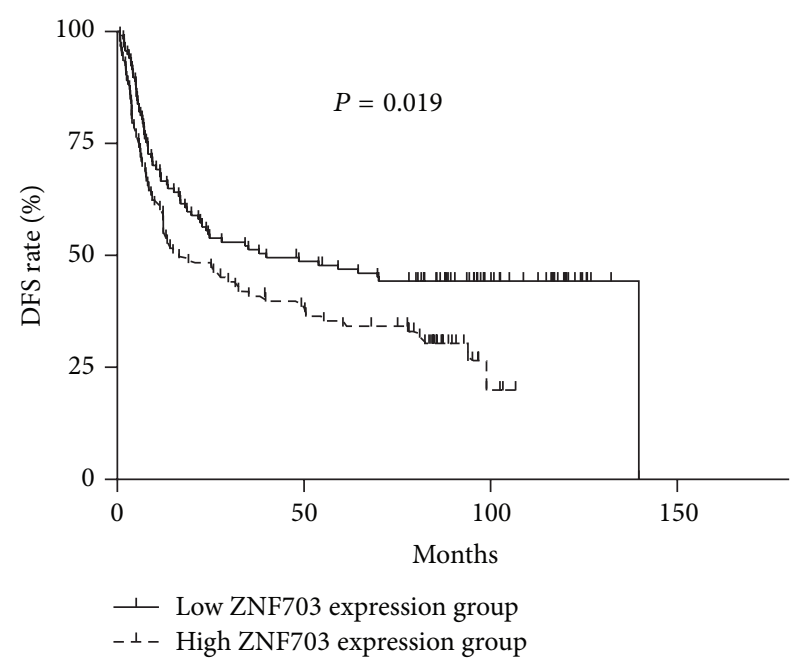

(b)

FIGURE 4: ZNF703 overexpression is associated with worse OS and DFS by Kaplan-Meier analysis.

\section{Authors' Contribution}

The first authors Hang Yang and Wen-Qi Jiang, contributed equally to this paper.

\section{Acknowledgments}

This work was supported by grants from The Scientific Research Foundation for the Returned Overseas Chinese Scholars of Sun Yat-Sen University Cancer Center and from the National High Technology Research and Development Program of China (863 Program) (no. 2012AA02A501). The authors thank all the researchers, including the physicians, pathologists, and technicians involved in this study.

\section{References}

[1] R. Siegel, J. Ma, Z. Zou, and A. Jemal, "Cancer statistics, 2014," CA: Cancer Journal for Clinicians, vol. 64, no. 1, pp. 9-29, 2014.

[2] A. Jemal, F. Bray, M. M. Center, J. Ferlay, E. Ward, and D. Forman, "Global cancer statistics," CA: A Cancer Journal for Clinicians, vol. 61, no. 2, pp. 69-90, 2011.

[3] B. Wang, S. Zhang, K. Yue, and X.-D. Wang, "The recurrence and survival of oral squamous cell carcinoma: a report of 275 cases," Chinese Journal of Cancer, vol. 32, no. 11, pp. 614-618, 2013.

[4] A. A. Forastiere, K. K. Ang, D. Brizel et al., "Head and neck cancers," Journal of the National Comprehensive Cancer Network, vol. 6, no. 7, pp. 646-695, 2008. 
[5] L. Mao, W. K. Hong, and V. A. Papadimitrakopoulou, "Focus on head and neck cancer," Cancer Cell, vol. 5, no. 4, pp. 311-316, 2004.

[6] J. D. Brown, S. Dutta, K. Bharti et al., "Expression profiling during ocular development identifies $2 \mathrm{Nlz}$ genes with a critical role in optic fissure closure," Proceedings of the National Academy of Sciences of the United States of America, vol. 106, no. 5, pp. 14621467, 2009.

[7] R. Dorfman, L. Glazer, U. Weihe, M. F. Wernet, and B.-Z. Shilo, "Elbow and Noc define a family of zinc finger proteins controlling morphogenesis of specific tracheal branches," Development, vol. 129, no. 15, pp. 3585-3596, 2002.

[8] E. M. Slorach, J. Chou, and Z. Werb, "Zeppol is a novel metastasis promoter that represses E-cadherin expression and regulates p120-catenin isoform expression and localization," Genes and Development, vol. 25, no. 5, pp. 471-484, 2011.

[9] D. G. Holland, A. Burleigh, A. Git et al., "ZNF703 is a common Luminal B breast cancer oncogene that differentially regulates luminal and basal progenitors in human mammary epithelium," EMBO Molecular Medicine, vol. 3, no. 3, pp. 167-180, 2011.

[10] F. Sircoulomb, N. Nicolas, A. Ferrari et al., "ZNF703 gene amplification at 8p12 specifies luminal B breast cancer," The EMBO Molecular Medicine, vol. 3, no. 3, pp. 153-166, 2011.

[11] I. Reynisdottir, A. Arason, B. O. Einarsdottir et al., "High expression of ZNF703 independent of amplification indicates worse prognosis in patients with luminal B breast cancer," Cancer Medicine, vol. 2, no. 4, pp. 437-446, 2013.

[12] X. Zhang, X. Mu, O. Huang et al., "Luminal breast cancer cell lines overexpressing ZNF703 are resistant to tamoxifen through activation of Akt/mTOR signaling," PLoS ONE, vol. 8, no. 8, Article ID e72053, 2013.

[13] G. Yang, F. Ma, M. Zhong et al., "ZNF703 acts as an oncogene that promotes progression in gastric cancer," Oncology Reports, vol. 31, no. 4, pp. 1877-1882, 2014.

[14] F. Ma, L. Bi, G. Yang et al., "ZNF703 promotes tumor cell proliferation and invasion and predicts poor prognosis in patients with colorectal cancer," Oncology Reports, vol. 32, no. 3, pp. 10711077, 2014.

[15] R. Fluss, D. Faraggi, and B. Reiser, "Estimation of the Youden index and its associated cutoff point," Biometrical Journal, vol. 47, no. 4, pp. 458-472, 2005.

[16] J. Califano, P. Van Der Riet, W. Westra et al., "Genetic progression model for head and neck cancer: implications for field cancerization," Cancer Research, vol. 56, no. 11, pp. 2488-2492, 1996.

[17] M. Partridge, G. Emilion, S. Pateromichelakis, E. Phillips, and J. Langdon, "Location of candidate tumour suppressor gene loci at chromosomes $3 p, 8 p$ and $9 p$ for oral squamous cell carcinomas," International Journal of Cancer, vol. 83, no. 3, pp. 318-325, 1999.

[18] S. B. Scholnick, B. H. Haughey, J. B. Sunwoo et al., "Chromosome 8 allelic loss and the outcome of patients with squamous cell carcinoma of the supraglottic larynx," Journal of the National Cancer Institute, vol. 88, no. 22, pp. 1676-1682, 1996.

[19] J. B. Sunwoo, M. S. Holt, D. M. Radford, C. Deeker, and S. B. Scholnick, "Evidence for multiple tumor suppressor genes on chromosome arm $8 \mathrm{p}$ in supraglottic laryngeal cancer," Genes Chromosomes and Cancer, vol. 16, no. 3, pp. 164-169, 1996.

[20] C. L. Wu, L. Roz, P. Sloan et al., "Deletion mapping defines three discrete areas of allelic imbalance on chromosome arm 8p in oral and oropharyngeal squamous cell carcinomas," Genes, Chromosomes and Cancer, vol. 20, no. 4, pp. 347-353, 1997.
[21] H. Ye, N. Pungpravat, B.-L. Huang et al., "Genomic assessments of the frequent loss of heterozygosity region on 8 p21.3 p22 in head and neck squamous cell carcinoma," Cancer Genetics and Cytogenetics, vol. 176, no. 2, pp. 100-106, 2007.

[22] M. J. Garcia, J. C. M. Pole, S.-F. Chin et al., "A 1 Mb minimal amplicon at 8p11-12 in breast cancer identifies new candidate oncogenes," Oncogene, vol. 24, no. 33, pp. 5235-5245, 2005.

[23] V. Gelsi-Boyer, B. Orsetti, N. Cervera et al., "Comprehensive profiling of 8p11-12 amplification in breast cancer," Molecular Cancer Research, vol. 3, no. 12, pp. 655-667, 2005.

[24] S. S. Kwek, R. Roy, H. Zhou et al., "Co-amplified genes at 8p12 and 11q13 in breast tumors cooperate with two major pathways in oncogenesis," Oncogene, vol. 28, no. 17, pp. 1892-1903, 2009.

[25] J. Adnane, P. Gaudray, C. A. Dionne et al., "BEK and FLG, two receptors to members of the FGF family, are amplified in subsets of human breast cancers," Oncogene, vol. 6, no. 4, pp. 659-663, 1991.

[26] A. Letessier, F. Sircoulomb, C. Ginestier et al., "Frequency, prognostic impact, and subtype association of 8p12, 8q24, 11q13, 12p13, 17q12, and 20q13 amplifications in breast cancers," BMC Cancer, vol. 6, article 245, 2006.

[27] Z. Q. Yang, K. L. Streicher, M. E. Ray, J. Abrams, and S. P. Ethier, "Multiple interacting oncogenes on the 8p11-p12 amplicon in human breast cancer," Cancer Research, vol. 66, no. 24, pp. 11632-11643, 2006.

[28] P. Spellman and J. Gray, "A new treasure in the breast cancer gene hunt," Nature Medicine, vol. 17, no. 4, pp. 422-423, 2011.

[29] C. Curtis, S. P. Shah, S. F. Chin et al., "The genomic and transcriptomic architecture of 2,000 breast tumours reveals novel subgroups," Nature, vol. 486, no. 7403, pp. 346-352, 2012.

[30] J. L. Andrews, A. C. Kim, and J. R. Hens, "The role and function of cadherins in the mammary gland," Breast Cancer Research, vol. 14, no. 1, p. 203, 2012. 


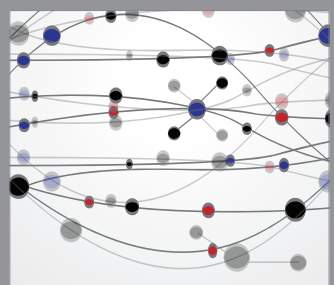

The Scientific World Journal
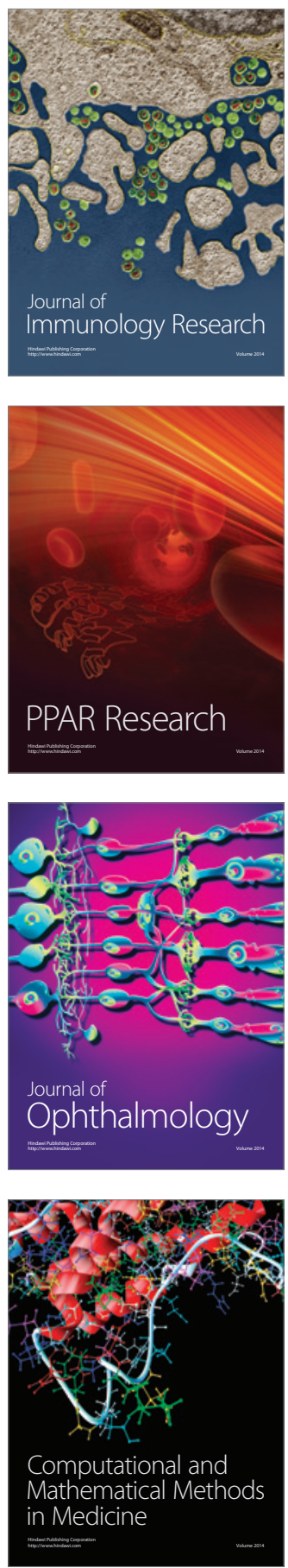

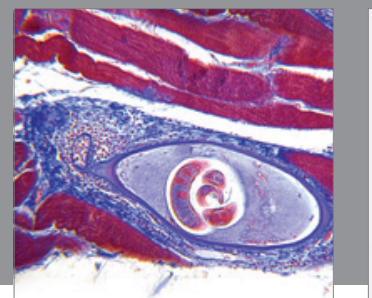

Gastroenterology

Research and Practice
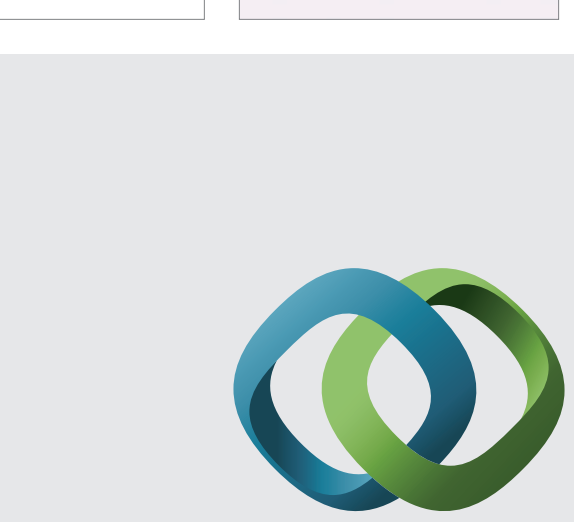

\section{Hindawi}

Submit your manuscripts at

http://www.hindawi.com
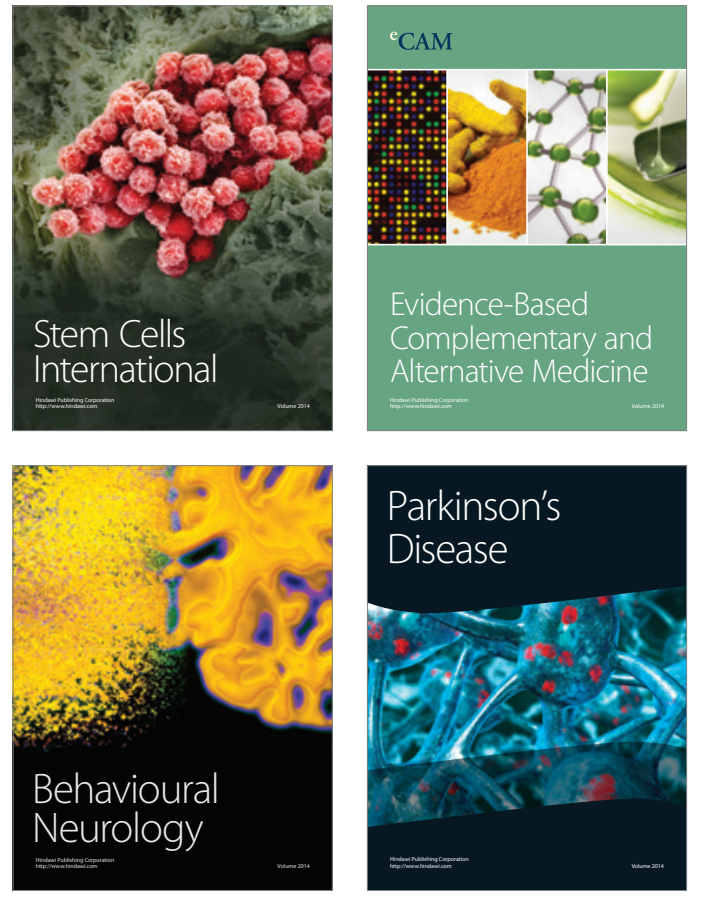
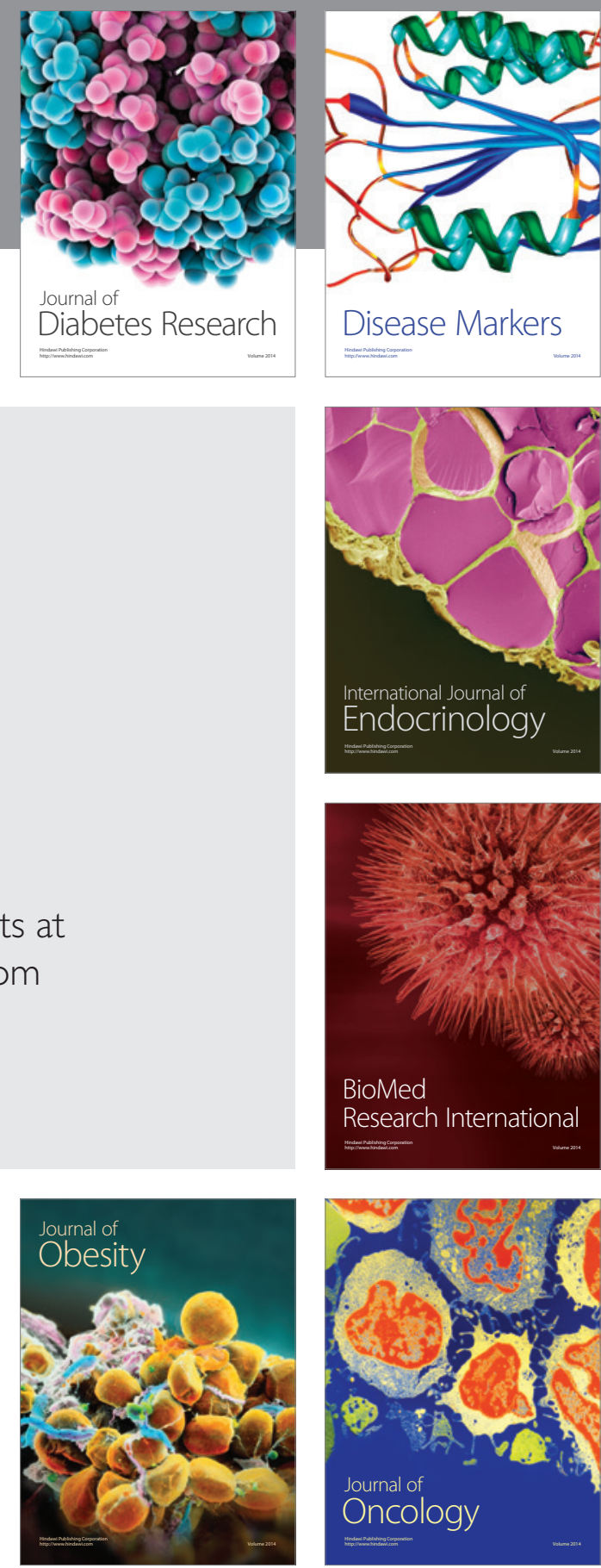

Disease Markers
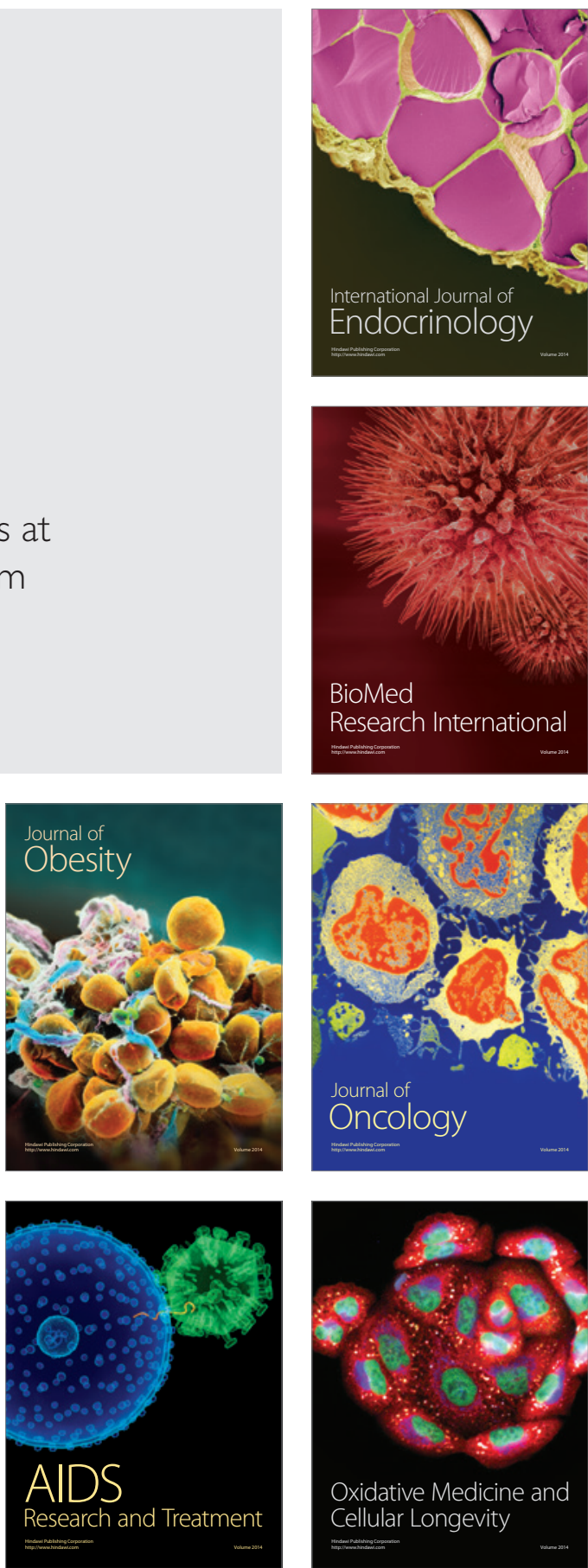